\title{
The diagnostic value of metagenomic next-generation sequencing for identifying Streptococcus pneumoniae in paediatric bacterial meningitis
}

Xi-xi Zhang ${ }^{1+}$, Ling-yun Guo ${ }^{1 \dagger}$, Lin-lin Liu', Ao Shen², Wen-ya Feng ${ }^{1}$, Wen-hua Huang ${ }^{3}$, Hui-li Hu ${ }^{1}$ Bing Hu', Xin Guo', Tian-ming Chen ${ }^{1}$, He-ying Chen ${ }^{1}$, Yong-qiang Jiang ${ }^{3}$ and Gang Liu ${ }^{1 *}$

\begin{abstract}
Background: There is currently no research on the diagnostic value of metagenomic next-generation sequencing (mNGS) for a single pathogens in CSF. The aim of this study was to analyse the value of mNGS for identifying Streptococcus pneumoniae (S. pneumoniae) in paediatric bacterial meningitis.

Methods: Bacterial meningitis (BM) cases from October 23, 2014, to December 31, 2016, and December 1, 2017, to July 31, 2018 at Beijing Children's Hospital were reviewed. Clinical features and pathogens were analysed.

Results: We diagnosed 135 patients with BM in this study. A total of 43 S. pneumoniae were identified by combination methods. 26/135 (19.3\%) patients had positive results in S. pneumoniae by blood and/or cerebrospinal fluid (CSF) culture. Alere BinaxNow ${ }^{\circledR}$ Streptococcus pneumoniae Antigen test was positive in 35/135(25.9\%) cases. 32/ 135 (23.7\%) S. pneumoniae were identified by mNGS. Six CSF samples were identified as S. pneumoniae only by mNGS technology. Taking culture as the gold standard, the sensitivity and specificity of mNGS for diagnosing $S$. pneumoniae meningitis were 73.1 and $88.1 \%$, respectively. The positive predictive value (PPV) and negative predictive value (NPV) of diagnosing S. pneumoniae meningitis by mNGS were 59.4 and $93.2 \%$, respectively. When comparison between mNGS and combined tests (culture and Alere BinaxNow ${ }^{\circledR}$ Streptococcus pneumoniae Antigen test), the sensitivity and specificity of mNGS for S. pneumoniae identification were 70.3 and 93.9\%, the PPV and NPV in the identification of S. pneumoniae by mNGS were 81.4 and $89.3 \%$, respectively. The difference in number of unique reads of S. pneumoniaein from CSF sample ( $<14$ days onset) and CSF sample ( $>14$ days from onset) was statistically significant (170.5 VS. 13, $P=0.019$ ). The difference in the collected time of CSF for culture and mNGS was statistically significant (4 days VS. 14 days, $P<0.001$ ).
\end{abstract}

Conclusions: mNGS has high sensitivity and specificity for S. pneumoniae identification. The pathogen load (number of unique reads) of S. pneumonia is related to the CSF collection time. mNGS was less affected than culture by the use of antibiotics before CSF collection.

Keywords: Metagenomic next-generation sequencing (mNGS), Sensitivity, Specificity, Streptococcus pneumonia, Meningitis, Children

\footnotetext{
* Correspondence: liugangbch@sina.com

${ }^{+}$Xi-xi Zhang and Ling-yun Guo contributed equally to this work.

${ }^{1}$ Key Laboratory of Major Diseases in Children, Ministry of Education,

Department of Infectious Diseases, Beijing Children's Hospital, Capital

Medical University, National Center for Children's Health, No. 56 Nan Lishi

Road, Beijing 100045, China

Full list of author information is available at the end of the article
}

(c) The Author(s). 2019 Open Access This article is distributed under the terms of the Creative Commons Attribution 4.0 International License (http://creativecommons.org/licenses/by/4.0/), which permits unrestricted use, distribution, and reproduction in any medium, provided you give appropriate credit to the original author(s) and the source, provide a link to the Creative Commons license, and indicate if changes were made. The Creative Commons Public Domain Dedication waiver (http://creativecommons.org/publicdomain/zero/1.0/) applies to the data made available in this article, unless otherwise stated. 


\section{Highlights}

Metagenomic Next-generation sequencing (mNGS) had high sensitivity and specificity for S. pneumoniae identification in CSF specimens.

mNGS was less affected than culture by the use of antibiotics before CSF collection.

The number of unique reads of $S$. pneumoniae detected by mNGS was related to the duration from onset to the sample collection time.

\section{Background}

Bacterial Meningitis (BM) is an infection of the meninges and subarachnoid space that can sometimes present as an infection of the brain cortex and parenchyma [1]. BM had an annual incidence of 16 million people worldwide in 2013, of which 1.6 million had sequelaes [2]. BM causes significant morbidity and mortality in both developed and developing countries [3-5]. The fatality rate of Streptococcus pneumoniae (S. pneumoniae) meningitis can reach $20-37 \%$ in high-income countries and up to $51 \%$ in low-income countries [6]. Neurological sequelae are common among survivors [7-10]. Therefore, prompt pathogen diagnosis and accurate antibiotic treatment are essential to improve the prognosis of BM.

With the application of the meningococcal vaccine, $S$. pneumoniae conjugate vaccine and Hib vaccine, the incidence of BM caused by these pathogens has decreased in developed countries [11]. However, the pneumococcal conjugate vaccine is not universally used in China. A multicentre study of BM in Chinese children showed that $S$. pneumoniae was still the most common pathogen, occurring in up to $46.5 \%$ of cases [12]. The conventional pathogen detection method (culture) may take a long time (3 to 5 days) and have a low positive rate. Metagenomic next-generation sequencing (mNGS) is a high-throughput sequencing method that can directly detect the nucleic acids of pathogens in clinical specimens and then analyse the nucleic acid sequences by bioinformatics methods. As a novel diagnostic tool, mNGS has been used for the identification of various pathogens such as bacteria, viruses, fungi and parasites from clinical samples (tissues, CSF or plasma) in an unbiased, simultaneous and direct manner [13-20].

However, at present, there are few studies on pathogen detection by mNGS in bacterial meningitis. Our team previously identified pathogens from cerebrospinal fluid specimens in children with bacterial meningitis by mNGS. The results showed that $\mathrm{mNGS}$ is of great value for the identification of pathogens in cerebrospinal fluid (CSF), and $S$. pneumoniae is still the most common pathogen [17]. However, there is currently no research on the diagnostic value of mNGS for a single pathogen. Hence, this study aimed to investigate the diagnostic value of mNGS for identifying S. pneumoniae in children with BM.

\section{Methods}

\section{Study population and specimen collection}

All BM patients aged 29 days to 18 years were admitted to the Department of Infectious Diseases at Beijing Children's Hospital from October 23, 2014, to December 31, 2016, and December 1, 2017, to July 31, 2018. The diagnosis criteria for $\mathrm{BM}$ were consistent with the World Health Organization (WHO), as follows: (1) acute fever (>38.5 C rectal or $>38.0 \mathrm{C}$ axillary); (2) headache, meningeal irritation, or altered consciousness; (3) at least one of the following in the cerebrospinal fluid: leukocytes $>100$ cells $/ \mathrm{mm}$ [3] or leukocytes 10-100 cells/mm [3] with elevated protein (> $100 \mathrm{mg} / \mathrm{dL})$ or decreased glucose $(<40 \mathrm{mg} / \mathrm{dL})$; and (4) positive culture, positive Gram stain, or positive bacterial antigen in the CSF. A case meeting diagnostic criteria 1, 2, and 3 at the same time was considered a probable case. A probable case meeting criterion 4 was considered a confirmed case. Exclusion criteria were as follows: cerebrospinal fluid $<1 \mathrm{ml}$ and bloody CSF. The clinical data of all included cases including demographic characteristics, antibiotic use, specimen collection and clinical microbiology tests results [culture and Alere BinaxNow ${ }^{\bullet}$ Streptococcus pneumoniae Antigen test (Alere, USA)] were recorded.

One millilitre of CSF was collected for mNGS when the lumbar puncture was performed after admission. This study was approved by the Ethics Committee of Beijing Children's Hospital affiliated to Capital Medical University (No. 2017-74). Written informed consent was obtained from the patient's parents or other legal representatives.

\section{DNA extraction}

DNA was extracted directly from the 300ul CSF sample (each patient and negative "no-template" control) using the TIANamp Micro DNA Kit (DP316, Tiangen Biotech, Beijing, China). The sample was added proteinase K (10 $\mathrm{ml}$ ) and $300 \mathrm{ml}$ buffer $\mathrm{GB}$ (with carrier RNA) and then incubated at $56{ }^{\circ} \mathrm{C}(10 \mathrm{~min})$. After $300 \mathrm{ul}$ cold absolute ethyl alcohol was added and the tube was incubated at room temperature $(5 \mathrm{~min})$. Transferring the liquid to a new adsorption column and the liquid was washed with buffer GD and buffer PW. The DNA was dissolved in 40ul of Tris-ethylenediaminetetraacetic acid buffer.

\section{Library generation, and sequencing}

The extracted DNA was sonicated with a Bioruptor Pico device to generate 200-300 bp fragments. According to the standard protocol of the BGISEQ-500 sequencing platform (BGI-Tianjin, Tianjin, China), DNA libraries were constructed through end repaired, adapter added overnight and polymerase chain reaction amplification to the extracted DNA. Quality control was carried out using a bioanalyser (Agilent 2100, Agilent Technologies, Santa Clara, CA, USA) combined with quantitative PCR 
to measure the adapters before sequencing. DNA sequencing was then performed using the BGISEQ-500 platform (BGI-Tianjin, Tianjin, China) [21].

\section{Data processing and analysis}

High quality sequencing data was generated after removal of short $(<35 \mathrm{bp})$ reads, low quality and low complexity reads. The readings were then mapped to the human reference genome (hg19 and $\mathrm{YH}$ sequences) using the Burrows-Wheeler Aligner [22]. The remaining data were aligned with the NCBI microbial genome database (ftp://ftp.ncbi.nlm.nih.gov/genomes/), which included the genome sequences of 3446 bacterial species (104 species of Mycobacterium tuberculosis and 45 species of mycoplasma/chlamydia), 1515 viral species, 206 fungal species and 140 parasites connected to human diseases. The mapped data were used for further analysis. The depth and coverage of each species was calculated using Soap Coverage on the SOAP website (http:// soap.genomics.org.cn/).

\section{Quantitative real-time PCR (qPCR) validation}

We performed $S$. pneumonia $\mathrm{qPCR}$ to validate the mNGS results. The DNA was extracted using the QIAamp cador Pathogen Mini Kit (Qiagen, 54,106). qPCR was carried out on the ViiATM 7 real-time PCR system using cycling conditions comprising $2 \mathrm{~min}$ at $50^{\circ} \mathrm{C}$ and $10 \mathrm{~min}$ at $95^{\circ} \mathrm{C}$ followed by 45 two-step cycles of $15 \mathrm{~min}$ at $95^{\circ} \mathrm{C}$ and $1 \mathrm{~min}$ at $60^{\circ} \mathrm{C}$. The sequences of the primers and probes are as follows: F: ACGCAATCTAGCAGATGAAGCA, R: TCGTGCGTTTTAATTCCAGCT and P: FAM-AACGCTTGATACAGGGAGMGB [23].

\section{Statistical methods}

Continuous variables were expressed as the mean \pm standard deviation or as the median. Two groups were compared using the independent t-test for parametric data and the Mann-Whitney $U$ test for non-parametric data. Continuous variables with $P$-values $<0.05$ were considered statistically significant, and all tests were 2tailed. Based on the extracted data, a $2 \times 2$ contingency table was used to determine sensitivity, specificity, PPV, and NPV. All of the statistical analyses were conducted using SPSS 23.0 software (SPSS Inc. USA).

\section{Results}

\section{Patient demographics and microbiology results}

A total of 135 children with BM were included in this study. S. pneumoniae was identified in $43 / 135$ (31.9\%) patients by all microbiology tests. A total of $26 / 43(60.5 \%)$ cases were male, and the median age was $11.5(8.5,48.9)$ months. A total of $37 / 135$ (27.4\%) cases were identified as $S$. pneumonia infection by clinical microbiology tests (culture and/or Alere BinaxNow ${ }^{\circ}$ Streptococcus pneumoniae Antigen test). S. pneumonia was identified by blood and/or CSF culture in 26/135 (19.3\%) cases. A total of 35/ 135 (25.9\%) patients had positive results on the Alere BinaxNow ${ }^{\circ}$ Streptococcus pneumoniae Antigen test. S. pneumoniae was identified in 32/135 (23.7\%) patient CSF specimens by mNGS. Six CSF samples were identified as S. pneumoniae only by mNGS.

\section{mNGS information and the related influencing factors}

Among 32 patients who were diagnosed with S. pneumoniae meningitis by $\mathrm{mNGS}$, the number of unique reads of $S$. pneumonia ranged from 4 to 341,303 . The coverage of $S$. pneumonia ranged from 0.011 to $86.68 \%$, with a depth value of 1-36.57 (Table 1). The collection time of CSF specimens for mNGS was 4 to 121 days after disease onset, and the median collection time was 14 days. The difference in the number of unique reads of $S$. pneumoniae in the different groups (CSF collection time $<14$ days and $>14$ days from onset) was statistically significant (170.5 vs. $13, P=$ 0.019) (Fig. 1). All $32 \mathrm{mNGS}$-positive children were treated with antibiotics prior to $\mathrm{mNGS}$ sample collection.

Based on the number of unique reads of S. pneumoniae, 32 patients were divided into two groups. The unique reads $\leq 100$ group comprised 17 (41.4\%) patients, while the unique reads $>100$ group comprised 15 patients. Comparing the two groups, patients in the unique reads $>100$ group had a generally shorter duration from disease onset to sample collection than patients in the mNGS unique reads $\leq 100$ group ( 11 days vs. 20 days, $P=0.027$ ). There was a significant difference in the simultaneous CSF white blood cell count and protein level between the two groups $\left(10 \times 10^{9} / \mathrm{L}\right.$ vs. $32 \times 10^{9} / \mathrm{L}, P=$ $0.001 ; 1280 \mathrm{mg} / \mathrm{L}$ vs. $855 \mathrm{mg} / \mathrm{L}, P=0.016$ ) (Table 2).

\section{Comparison of mNGS and clinical microbiology methods}

The median collection time of CSF specimens for culture that had positive $S$. pneumoniae results was $4(3,5)$ days. The median collection time of CSF specimens for mNGS that had positive S. pneumoniae results was 14 $(8,23)$ days from onset. The difference in the collected time of CSF for culture and mNGS was statistically significant ( 4 days vs. 14 days, $P<0.001$ ).

Among the $135 \mathrm{BM}$ patients, mNGS and clinical microbiology tests (culture and/or Alere BinaxNow ${ }^{\circ}$ Streptococcus pneumoniae Antigen test) were both positive for S. pneumonia in $26(19.3 \%)$ samples and were both negative for S. pneumonia in $92(68.1 \%)$ samples. The accordance rate of mNGS and clinical microbiology tests (culture and/or Alere BinaxNow ${ }^{\circ}$ Streptococcus pneumoniae Antigen test) for the identification of $S$. pneumoniae was $87.4 \%(118 / 135)$.

The comparison between mNGS and clinical microbiology tests (culture and Alere BinaxNow ${ }^{\circ}$ Streptococcus 
Table 1 Clinical microbiology results and sequencing information for patients who were positive for S. pneumonia by mNGS

\begin{tabular}{|c|c|c|c|c|c|c|c|c|}
\hline \multirow[b]{2}{*}{ Patient } & \multicolumn{3}{|c|}{ Clinical microbiology tests } & \multicolumn{4}{|l|}{$\mathrm{mNGS}$} & \multirow[t]{2}{*}{$\mathrm{qPCR}$} \\
\hline & Pathogen & Culture (blood/CSF) & Antigen test & Pathogen & Reads & Coverage $\%$ & Depth & \\
\hline $\mathrm{P} 1$ & SP & - & + & $\mathrm{SP}$ & 262 & 1.1 & 1 & ND \\
\hline P2 & SP & + & + & SP & 1105 & 11 & 1.1 & ND \\
\hline P3 & SP & + & + & $\mathrm{SP}$ & 573 & 2.6 & 1 & + \\
\hline P4 & SP & + & + & $\mathrm{SP}$ & 429 & 1.9 & 1 & + \\
\hline P5 & SP & - & + & $\mathrm{SP}$ & 425 & 1.9 & 1 & + \\
\hline P6 & SP & - & + & SP & 176 & 1.8 & 1 & ND \\
\hline P7 & SP & + & ND & $\mathrm{SP}$ & 162 & 0.75 & 1 & + \\
\hline P8 & SP & + & + & SP & 38 & 0.16 & 1 & ND \\
\hline P9 & SP & - & + & $\mathrm{SP}$ & 21 & 0.12 & 1 & ND \\
\hline P10 & SP & + & + & $S P$ & 13 & 0.066 & 1 & ND \\
\hline P11 & $\mathrm{SP}$ & + & + & $\mathrm{SP}$ & 11 & 0.052 & 1 & $\mathrm{ND}$ \\
\hline P12 & SP & + & + & $\mathrm{SP}$ & 7 & 0.048 & 1 & - \\
\hline P13 & SP & + & + & $\mathrm{SP}$ & 6 & 0.034 & 1 & - \\
\hline P14 & SP & + & + & SP & 4 & 0.041 & 1 & ND \\
\hline P15 & SP & - & + & $\mathrm{SP}$ & 4 & 0.016 & 1 & - \\
\hline P16 & $\mathrm{SP}$ & - & + & $\mathrm{SP}$ & 3189 & 28 & 1.3 & ND \\
\hline P17 & $\mathrm{SP}$ & - & + & $\mathrm{SP}$ & 23,247 & 75 & 3.9 & ND \\
\hline P18 & SP & + & + & $\mathrm{SP}$ & 25 & 0.19 & 1 & + \\
\hline P19 & SP & + & + & $\mathrm{SP}$ & 2603 & 7.25 & 1.06 & ND \\
\hline P20 & SP & + & + & $\mathrm{SP}$ & 341,303 & 86.68 & 36.57 & + \\
\hline P21 & SP & + & + & $\mathrm{SP}$ & 165 & 0.7413 & 1.01 & + \\
\hline P22 & $\mathrm{SP}$ & + & + & $\mathrm{SP}$ & 1335 & 12.03 & 1.09 & + \\
\hline P23 & SP & + & + & $\mathrm{SP}$ & 456 & 5.41 & 1.04 & + \\
\hline P24 & $\mathrm{SP}$ & + & + & SP & 32 & 0.0744 & 1 & ND \\
\hline P25 & $\mathrm{SP}$ & + & + & $\mathrm{SP}$ & 218 & 1.04 & 1 & ND \\
\hline P26 & $\mathrm{SP}$ & + & + & $\mathrm{SP}$ & 20 & 0.1488 & 1 & - \\
\hline P27 & - & - & - & $\mathrm{SP}$ & 33 & 0.31 & 1 & ND \\
\hline P28 & SA & SA & ND & $\mathrm{SP}$ & 27 & 0.15 & 1 & - \\
\hline P29 & - & - & - & $\mathrm{SP}$ & 24 & 0.12 & 1 & ND \\
\hline P30 & - & - & ND & $\mathrm{SP}$ & 12 & 0.95 & 1 & ND \\
\hline P31 & - & - & - & SP & 6 & 0.055 & 1 & ND \\
\hline P32 & - & - & - & $S P$ & 4 & 0.011 & 1 & - \\
\hline
\end{tabular}

Note: $m$ NGS metagenomic next-generation sequencing, CSF Cerebrospinal fluid, qPCR quantitative real-time PCR, SP Streptococcus pneumonia, SA Streptococcus aureus, E. coli Escherichia coli, + positive, - negative, ND Not done

pneumoniae Antigen test) was shown in Table 3. Taking culture as the gold standard, the sensitivity and specificity of $S$. pneumoniae identification by mNGS were73.1 and $88.1 \%$, respectively. The PPV and NPV of diagnosing $S$. pneumoniae meningitis by mNGS were 59.4 and $93.2 \%$, respectively. Comparing mNGS and the combined tests (culture and Alere BinaxNow ${ }^{\circ}$ Streptococcus pneumoniae Antigen test), the sensitivity and specificity of mNGS for S. pneumoniae identification were70.3 and 93.9\%, respectively, and the PPV and NPV for the identification of $S$. pneumoniae by mNGS were 81.4 and $89.3 \%$, respectively.
In total, we performed qPCR validation for 15 CSF specimens, while insufficient CSF sample was available for $\mathrm{qPCR}$ validation in the other cases. These $15 \mathrm{CSF}$ specimens were collected for mNGS and qPCR at the same time. The qPCR results were positive in $9(9 / 15$, $60 \%)$ cases [(mNGS unique reads: $429(25-341,303)]$ and negative in $6(6 / 15,40 \%)$ cases. The latter 6 cases had fewer unique reads of $S$. pneumonia, and the number of unique reads was 4, 4, 6, 7, 20 and 27. The clinical microbiology tests (culture and/or Alere BinaxNow ${ }^{\circ}$ Streptococcus pneumoniae Antigen test) were positive 


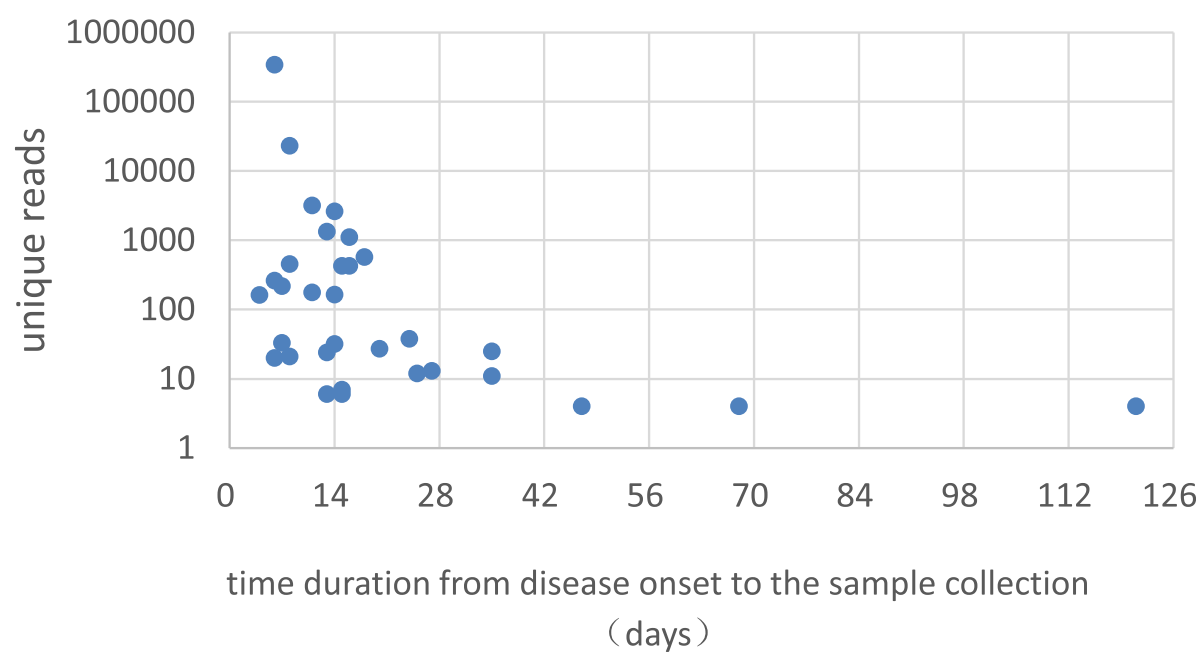

Fig. 1 The relationship between unique reads of Streptococcus pneumonia and sample collection time

Table 2 Comparison of different mNGS unique read groups

\begin{tabular}{|c|c|c|c|}
\hline Items & $\begin{array}{l}m N G S \text { unique reads } \leq 100 \\
(N=17)\end{array}$ & $\begin{array}{l}\text { mNGS unique reads }>100 \\
(N=15)\end{array}$ & $P$ \\
\hline Sex (female/male) & $12 / 5$ & $7 / 8$ & 0.169 \\
\hline Age (months) & $11.2(5.9,33.5)$ & $13.9(9.2,48.9)$ & 0.290 \\
\hline Duration from onset to CSF collection (days) & $20(13,35)$ & $11(7,16)$ & 0.027 \\
\hline Fever [n (\%)] & $17(100)$ & $15(100)$ & \\
\hline Seizure $[n(\%)]$ & $8(47.1)$ & $10(66.7)$ & 0.265 \\
\hline Vomiting [n (\%)] & 10(58.8) & $9(60)$ & 0.946 \\
\hline Meningeal irritation [n (\%)] & $9(52.9)$ & $12(80)$ & 0.103 \\
\hline Altered mental status [n (\%)] & $7(41.2)$ & $8(53.3)$ & 0.492 \\
\hline \multicolumn{4}{|l|}{ Peripheral blood } \\
\hline WBC $\left(\times 10^{9} / \mathrm{L}\right)$ & $16.98(15.10,23.69)$ & $17.3(11.9,24,2)$ & 0.895 \\
\hline Neutrophils (\%) & $78.2(60.7,86.3)$ & $77.8(62.7,85.6)$ & 0.985 \\
\hline Haemoglobin (g/L) & $104.4 \pm 19.0$ & $105.5 \pm 18.7$ & 0.767 \\
\hline C-reactive protein (mg/L) & 63.5(19.6104) & $109.5(49,157.4)$ & 0.281 \\
\hline \multicolumn{4}{|l|}{1 st $\mathrm{CSF}^{\mathrm{a}}$} \\
\hline WBC $\left(\times 10^{6} / \mathrm{L}\right)$ & $1714(495,2635)$ & $1886(910,3600)$ & 0.597 \\
\hline Protein (mg/L) & $1280(779,2250)$ & $1650(1161,3033)$ & 0.180 \\
\hline Glucose (mmol/L) & $1.84(1.00,3.34)$ & $0.67(0.19,1.92)$ & 0.081 \\
\hline \multicolumn{4}{|l|}{ Simultaneous CSF } \\
\hline WBC $\left(\times 10^{6} / \mathrm{L}\right)$ & $10(2,12)$ & $32(14,76)$ & 0.001 \\
\hline Protein (mg/L) & $1280(779,2250)$ & $855(527,1488)$ & 0.016 \\
\hline Glucose (mmol/L) & $3.42 \pm 0.99$ & $3.39 \pm 1.12$ & 0.928 \\
\hline
\end{tabular}

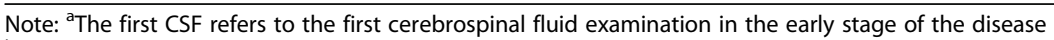

${ }^{b}$ Simultaneous CSF refers to the CSF results by clinical testing at which time CSF was also tested by mNGS

$P$-values in bold italic shows variables with evidence of association in univariate analysis 
Table 3 Diagnostic performance of mNGS compared with culture and Alere BinaxNow ${ }^{\circledast}$ Streptococcus pneumoniae Antigen test for the detection of Streptococcus pneumoniae

\begin{tabular}{lclll}
\hline $\begin{array}{l}\text { Clinical } \\
\text { microbiology } \\
\text { tests }\end{array}$ & mNGS & & \\
\cline { 2 - 5 } & Sensitivity (\%) & Specificity (\%) & PPV (\%) & NPV (\%) \\
\hline Culture $^{\text {a }}$ & 73.1 & 88.1 & 59.4 & 93.2 \\
Combined test $^{\text {b }}$ & 70.3 & 93.9 & 81.3 & 89.3 \\
\hline
\end{tabular}

Note: ${ }^{a}$ refers to the blood and/or CSF culture; ${ }^{b}$ refers to culture and or Alere BinaxNow ${ }^{\oplus}$ Streptococcus pneumoniae Antigen test. PPV positive predictive value, $N P V$ negative predictive value

for $S$. pneumonia in $4(4 / 6)$ cases (unique reads: $4,6,7$ and 20). One (1/6) case (unique reads: 4) was negative for $S$. pneumonia in the clinical microbiology tests (blood and/or CSF culture and Alere BinaxNow ${ }^{\oplus}$ Streptococcus pneumoniae Antigen test). Another case (1/6) (P28, unique reads: 27) was positive for Staphylococcus aureus ( $S$. aureus) in the blood culture. However, the result of specific PCR from CSF specimens was negative for both S. pneumoniae and S. aureus in P28.

In addition, we identified multiple pathogens in the CSF sample of P14 by mNGS, including Escherichia coli (E. coli) (unique reads 30 ) and $S$. pneumoniae (unique reads 4). In terms of clinical microbiology, P14 had a positive result for $S$. pneumoniae in the CSF culture and a positive Alere BinaxNow ${ }^{\oplus}$ Streptococcus pneumoniae Antigen test in the CSF. However, the Sanger validation of the CSF sample was negative for both S. pneumoniae and E. coli.

\section{Discussion}

In this study, clinical microbiology tests (culture and Alere BinaxNow ${ }^{\circledR}$ Streptococcus pneumoniae Antigen test) were compared with an emerging pathogen detection method (mNGS). When the combined culture and pneumococcal antigen test were regarded as the gold standard, mNGS showed high sensitivity and specificity for the diagnosis of $S$. pneumonia meningitis. Previous studies have reported that mNGS has different sensitivities and specificities for the identification of different types of pathogens (bacterial, viral or fungal). The sensitivity and specificity of mNGS for the identification of bacteria had a wide range, with sensitivity ranging from 50.7 to $100 \%$ and specificity ranging from 76.5 to $87.5 \%$ $[16,24]$. In this study, some of the CSF specimens were collected at the convalescence stage. If all CSF specimens were collected during the acute phase, the sensitivity of mNGS would be higher.

The number of unique reads of $S$. pneumoniae from CSF collected $\leq 14$ days from the disease onset was significantly higher than those from CSF collected > 14 days from the disease onset. Patients in the unique reads $>100$ group had a generally shorter duration from disease onset to the sample collection than those in the unique reads $\leq 100$ group. These results together suggest that the number of unique reads of S. pneumoniae detected by mNGS was associated with specimen collection time, which is consistent with a previous study showing that with the use of effective antibiotics and disease improvement, the number of unique reads of pathogens decreased [25].

In this study, we observed that the time from disease onset to CSF collection was longer for mNGS than for culture. All patients were given antibiotics prior to mNGS and culture, and all patients were treated with antibiotics after the onset of disease. This phenomenon suggested that after antibiotic use, the timespan for positive pathogen identification by mNGS was longer than the timespan for positive pathogen identification by culture. A previous study suggested that the DNA of the pathogen may survive in body fluids for a prolonged period of time, and mNGS was less affected than culture by the use of antibiotics before CSF collection [24, 26, 27].

Among the $6 \mathrm{CSF}$ samples that were negative for $S$. pneumonia by qPCR, S. pneumonia was identified in 4 (4/6) samples by both mNGS and clinical microbiology tests (blood and/or CSF culture and Alere BinaxNow ${ }^{\circ}$ Streptococcus pneumoniae Antigen test). This result showed that mNGS appears to have a higher sensitivity than qPCR in the test cases. S. pneumonia (unique reads 27) was identified in P28 CSF sample by mNGS, while $S$. aureus was detected by blood culture. The P28 CSF sample was found to be negative for both $S$. pneumoniae and $S$. aureus by qPCR. In many mNGS systems, short read sequences lead to difficulties in assembling and matching to the reference sequence, especially in repeat regions, resulting in difficulties defining the final pathogen. Therefore, when the number of unique reads is very small, the interpretation of pathogen data needs to be very careful and cautious [28].

We compared groups with $\leq 100$ unique reads and groups with $>100$ unique reads and analysed factors that could affect the number of unique reads of $S$. pneumoniae. There were significant differences in the simultaneous CSF white blood cell counts and protein levels between the two groups. These results suggested that the inflammation status during simultaneous mNGS detection might determine the number of unique reads of the pathogen. The number of unique reads may indirectly monitor disease progression.

Currently, the criteria for diagnosing single pathogens by mNGS are unclear. Different studies have reported different diagnostic criteria, mainly based on the coverage rate, the relative abundance of pathogens or unique reads of pathogens $[16,29]$.

In this study, the minimum number of unique reads of S. pneumoniae was 4 , and the clinical microbiology test was also positive for $S$. pneumoniae. Therefore, the positive criteria for single pathogen identification by mNGS testing require further research by a larger sample size.

There were several limitations in this study. First, the collection time of some CSF samples for mNGS and 
clinical microbiology tests was different. The collection time of CSF was later for mNGS than for the clinical microbiology tests, which may affect the positive identification rate of mNGS. Some of the CSF specimens were collected at the convalescence stage. Second, this study was retrospective and had a limited number of BM cases, and thus large-scale research is needed. Third, mNGS still has low sequencing depth, and the pathogen database is imperfect [30]. However, sequencing technology is developing rapidly, and it is believed that the depth of sequencing will improve within a short period of time. mNGS can further improve the sensitivity and specificity of pathogen identification [31].

\section{Conclusion}

This study is the first to analyse the diagnostic value of mNGS for the identification of a single pathogen $(S$. pneumoniae). mNGS has high sensitivity and specificity for S. pneumoniae identification. Our study also demonstrated the relationship between the pathogen load (unique reads) and CSF collection time. Although there are no uniform criteria for pathogen identification by mNGS, there are many difficulties in interpreting the mNGS results. With the development of mNGS technology, mNGS could be a promising alternative diagnostic tool for pathogen detection.

\section{Abbreviations}

BM: bacterial meningitis; CSF: cerebrospinal fluid; mNGS: metagenomic nextgeneration sequencing; NPV: negative predictive value; PPV: positive predictive value; qPCR: Quantitative real-time PCR; $S$.

pneumoniae: Streptococcus pneumonia; SA: Staphylococcus aureus

\section{Acknowledgements}

We would like to thank the Bio bank for Diseases in Children at Beijing Children's Hospital, Capital Medical University for their contribution of storing the CSF samples with standardized procedures.

\section{Author's contributions}

All of the authors had access to the full dataset (including the statistical reports and tables) and take responsibility for the integrity of the data and the accuracy of the data analysis. XXZ, LYG and GL conceived and designed the study. XXZ, LYG, LLL, WYF, BH, HLH, TMC, XG, and HYC were involved in the case and sample collection, analysis, and interpretation of the data. XXZ, LYG, AS, WHH and YQJ participated in the laboratory analysis of the CSF. XXZ wrote the first draft of the paper. XXZ, LYG and GL reviewed and approved the final report. All authors have read and approved the final manuscript.

\section{Funding}

This work was supported by The Special Fund of The Pediatric Medical Coordinated Development Center of Beijing Municipal Administration (No. XTZD20180501), Beijing Hospital Authority "Dengfeng" Talent Training Plan (DFL 20181201), and the National Science and Technology Major Project of China (No.2018ZX10305409). The funders had no role in study design, data collection and analysis, or preparation of the manuscript.

\section{Availability of data and materials}

The datasets analysed during the current study are available from the corresponding author on reasonable request.

\section{Ethics approval and consent to participate}

This study was reviewed and approved by the Ethics Committee of Beijing Children's Hospital Affiliated to Capital Medical University (2017-k-83). Informed consent was waived because this was a retrospectively study. We obtained patient data from the Medical Records and Statistics Room. We analysed the data anonymously. The raw data were permitted by the Ethics Committee of Beijing Children's Hospital Affiliated to Capital Medical University.

\section{Consent for publication}

Not applicable.

\section{Competing interests}

The authors declare that they have no competing interests.

\section{Author details}

${ }^{1}$ Key Laboratory of Major Diseases in Children, Ministry of Education, Department of Infectious Diseases, Beijing Children's Hospital, Capital Medical University, National Center for Children's Health, No. 56 Nan Lishi Road, Beijing 100045, China. ${ }^{2}$ Tianjin Medical Laboratory, BGl-Tianjin, Tianjin, China. ${ }^{3}$ State Key Laboratory of Pathogen and Biosecurity, Institute of Microbiology and Epidemiology, Academy of Military Medical Science, Beijing 100071, China.

Received: 27 February 2019 Accepted: 24 May 2019

Published online: 04 June 2019

\section{References}

1. van de Beek D, Brouwer M, Hasbun R, Koedel U, Whitney CG, Wijdicks E. Community-acquired bacterial meningitis. Nat Rev Dis Primers. 2016;2:16074.

2. Global Burden of Disease Study 2013 Collaborators. Global, regional, and national incidence, prevalence, and years lived with disability for 301 acute and chronic diseases and injuries in 188 countries, 1990-2013: a systematic analysis for the Global Burden of Disease Study 2013. Lancet. 2015;386(9995):743-800.

3. Lucas MJ, Brouwer MC, van de Beek D. Neurological sequelae of bacterial meningitis. J Inf Secur. 2016;73(1):18-27.

4. Mclntyre PB, O'Brien KL, Greenwood B, van de Beek D. Effect of vaccines on bacterial meningitis worldwide. Lancet. 2012;380(9854):1703-11.

5. Li Y, Yin Z, Shao Z, Li M, Liang X, Sandhu HS, et al. Acute meningitis and encephalitis syndrome study group. Population-based surveillance for bacterial meningitis in China, September 2006-December 2009. Emerg Infect Dis. 2014;20:61-9.

6. Brouwer MC, Tunkel AR, van de Beek D. Epidemiology, diagnosis, and antimicrobial treatment of acute bacterial meningitis. Clin Microbiol Rev. 2010;23(3):467-92.

7. Hupp S, Ribes S, Seele J, Bischoff C, Förtsch C, Maier E, et al. Magnesium therapy improves outcome in Streptococcus pneumoniae meningitis by altering pneumolysin pore formation. Br J Pharmacol. 2017;174(23):4295-307.

8. Grimwood K. Legacy of bacterial meningitis in infancy. many children continue to suffer functionally important deficits Br Med J. 2001;323(7321):523-4.

9. Libster R, Edwards KM, Levent F, Edwards MS, Rench MA, Castagnini LA, et al. Longterm outcomes of group B streptococcal meningitis. Pediatrics. 2012;130(1):e8.

10. Stockmann C, Ampofo K, Byington CL, Filloux F, Hersh AL, Blaschke AJ, et al. Pneumococcal meningitis in children: epidemiology, serotypes, and outcomes from 1997-2010 in Utah. Pediatrics. 2013;132(3):421-8.

11. Chávez-Bueno S, McCracken GH. Bacterial meningitis in children. Pediatr Clin N Am. 2005:52(3):795-810.

12. Li C, Feng WY, Lin AW, Zheng G, Wang YC, Han YJ, et al. Clinical characteristics and etiology of bacterial meningitis in Chinese children $>28$ days of age, January 2014-December 2016: a multicenter retrospective study. Int J Infect Dis. 2018;74:47-53.

13. Yao M, Zhou J, Zhu Y, Zhang Y, Lv X, Sun R, et al. Detection of listeria monocytogenes in CSF from three patients with meningoencephalitis by next-generation sequencing. J Clin Neurol. 2016;12(4):446-51.

14. Yu XJ, Liang MF, Zhang SY, Liu Y, Li JD, Sun YL, et al. Fever with thrombocytopenia associated with a novel bunyavirus in China. N Engl J Med. 2011;364(16):1523-32.

15. Guo LY, Feng WY, Guo X, Liu B, Liu G, Dong J. The advantages of nextgeneration sequencing technology in the detection of different sources of abscess. J Inf Secur. 2018;8 pii: S0163-4453(18)30245-7.

16. Li H, Gao H, Meng H, Wang Q, Li S, Chen H. Et al. detection of pulmonary infectious pathogens from lung biopsy tissues by metagenomic nextgeneration sequencing. Front Cell Infect Microbiol. 2018;8:205. 
17. Guo LY, Li YJ, Liu LL, Wu HL, Zhou JL, Zhang Y, et al. Detection of pediatric bacterial meningitis pathogens from cerebrospinal fluid by next-generation sequencing technology. J Inf Secur. 2018;11 pii: S0163-4453(18)30357-8.

18. Fan S, Qiao X, Liu L, Wu H, Zhou J, Sun R, et al. Next-generation sequencing of cerebrospinal fluid for the diagnosis of Neurocysticercosis. Front Neurol. 2018;9:471.

19. Brown JR, Bharucha T, Breuer J. Encephalitis diagnosis using metagenomics: application of next generation sequencing for undiagnosed cases. J Inf Secur. 2018;76(3):225-40.

20. Fan S, Ren H, Wei Y, Mao C, Ma Z, Zhang L, et al. Next-generation sequencing of cerebrospinal fluid for the diagnosis of Neurobrucellosis. Int $J$ Infect Dis. 2018;67:20-4.

21. Fang C, Zhong H, Lin Y, Chen B, Han M, Ren $\mathrm{H}$, et al. Assessment of the CPAS-based BGISEQ-500 platform for metagenomic sequencing. Gigascience. 2018;7(3):1-8.

22. Li H, Durbin R. Fast and accurate short read alignment with burrowswheeler transform. Bioinformatics. 2009;25(14):1754-60

23. Zhang C, Zheng X, Zhao C, Li Y, Chen S, Liu G, et al. Detection of pathogenic microorganisms from bloodstream infection specimens using TaqMan array card technology. Sci Rep. 2018;8(1):12828.

24. Miao Q, Ma Y, Wang Q, Pan J, Zhang Y, Jin W, et al. Microbiological diagnostic performance of metagenomic next-generation sequencing when applied to clinical practice. Clin Infect Dis. 2018;67(suppl_2):S231-40.

25. Ai JW, Zhang HC, Cui P, Xu B, Gao Y, Cheng Q, et al. Dynamic and direct pathogen load surveillance to monitor disease progression and therapeutic efficacy in central nervous system infection using a novel semiquantitive sequencing platform. J Inf Secur. 2018;76(3):307-10.

26. Gosiewski T, Ludwig-Galezowska AH, Huminska K, Sroka-Oleksiak A, Radkowski P, Salamon D, et al. Comprehensive detection and identification of bacterial DNA in the blood of patients with sepsis and healthy volunteers using next-generation sequencing method-the observation of DNAemia. Eur J Clin Microbiol Infect Dis. 2017;36(2):329-36.

27. Rhodes J, Hyder JA, Peruski LF, Fisher C, Jorakate P, Kaewpan A, et al. Antibiotic use in Thailand: quantifying impact on blood culture yield and estimates of pneumococcal bacteremia incidence. Am J Trop Med Hyg. 2010;83(2):301-6.

28. Hui P. Next generation sequencing: chemistry, technology and applications. Top Curr Chem. 2014:336:1-18

29. Langelier C, Zinter MS, Kalantar K, et al. Metagenomic sequencing detects respiratory pathogens in hematopoietic cellular transplant patients. Am J Respir Crit Care Med. 2018;197(4):524-8.

30. Gargis AS, Kalman L, Lubin IM. Assuring the quality of next-generation sequencing in clinical microbiology and public health laboratories. J Clin Microbiol. 2016;54(12):2857-65.

31. Thorburn F, Bennett S, Modha S, Murdoch D, Gunson R, Murcia PR, et al. The use of next generation sequencing in the diagnosis and typing of respiratory infections. J Clin Virol. 2015;69:96-100.

\section{Publisher's Note}

Springer Nature remains neutral with regard to jurisdictional claims in published maps and institutional affiliations.

Ready to submit your research? Choose BMC and benefit from:

- fast, convenient online submission

- thorough peer review by experienced researchers in your field

- rapid publication on acceptance

- support for research data, including large and complex data types

- gold Open Access which fosters wider collaboration and increased citations

- maximum visibility for your research: over $100 \mathrm{M}$ website views per year

At $\mathrm{BMC}$, research is always in progress.

Learn more biomedcentral.com/submissions 